\title{
Object-based representations govern both the storage of information in visual short-term memory and the retrieval of information from it
}

\author{
Philip T. Quinlan • Dale J. Cohen
}

Published online: 15 February 2011

(C) Psychonomic Society, Inc. 2011

\begin{abstract}
Interest is growing in how information is retained in visual short-term memory (VSTM). We describe an experiment that assesses VSTM within the context of multidimensional signal detection theory. On every trial, participants were presented with a 250 -ms display containing four colored shapes. They were then probed $900 \mathrm{~ms}$ later with a colored shape and made separate old/new judgments about the color and the shape. In any particular trial, one, both, or neither of the probed features had been presented. Performance differed according to whether both probed features belonged to a single object or to two different objects. When both probed features belonged to the same object, featural retrieval was better than would be predicted by independent feature storage. When both probed features belonged to two different objects, featural retrieval was worse than would be predicted by independent feature storage. We conclude that storage in and retrieval from VSTM operate on the basis of object-based representations.
\end{abstract}

Keywords Visual short-term memory. Object-based representations $\cdot$ Multidimensional signal detection theory

Recently, there has been increasing interest in the nature of visual short-term memory (VSTM). Two competing classes of theories have emerged: one that assumes that the

P. T. Quinlan $(\square)$

Department of Psychology, University of York,

Heslington,

York YO10 5DD, UK

e-mail: ptq1@york.ac.uk

D. J. Cohen

University of North Carolina,

Wilmington, NC, USA information stored in VSTM is object-based, and a second that assumes that the information stored in VSTM is feature-based. Here, we use a multidimensional signal detection procedure to directly assess predictions about featural independence that distinguish the two theories.

Much of the interest in VSTM can be traced back to the experiments reported by Luck and Vogel (1997) on performance in change detection tasks. On a typical trial, a sample array containing several to-be-remembered items was presented briefly, followed after a delay by a test array. The participants' task was to judge whether the test and sample arrays were identical. In the first experiment, the tobe-remembered items were randomly positioned colored squares and the number of items across the arrays varied. When the critical difference between the sample and test arrays was defined as a color change in one item, performance was nearly perfect for arrays containing up to four items. Performance decreased monotonically as the array set size increased further. Because additional verbal memory load failed to influence performance, Luck and Vogel concluded that this task was tapping into a capacity-limited VSTM.

Luck and Vogel (1997) used oriented colored bars to assess whether color and orientation are stored in VSTM independently or are integrated into object-based representations. In the orientation condition, participants were instructed to judge whether a change in the orientation of one bar occurred. In the color condition, they were to judge whether the color of one bar had changed. Finally, in the conjunction condition, they were instructed that either the color or the orientation of one bar could change. Importantly, all participants were equally accurate in their judgments of change, regardless of whether the change was carried on one featural dimension or two. Luck and Vogel concluded that "visual working memory stores integrated object percepts rather than individual features" (p.280). 
By this view, VSTM comprises a limited number of "slots," each of them capable of holding information about one object (Luck \& Vogel, 1997; Vogel, Woodman, \& Luck, 2001; Zhang \& Luck, 2008). According to this theory, the retention of the constituent features of an object does not require attentional resources: The representation of a given object is captured by the feature bindings that are present in a given slot in the store. We term this the visual object store theory.

Subsequently, alternative conclusions have been considered about the performance in such tasks. For instance, Wheeler and Treisman (2002) questioned the claim "that bound objects are the default units of storage" (p.60) and expressed doubts that feature bindings are stored at all. On the basis of evidence garnered from a range of change detection experiments, Wheeler and Treisman proposed that independent feature stores exist and that focused attention is required to bind an object's constituent features. Once they are bound, attention is then required to maintain the bindings in VSTM. We term this the visual feature store theory.

The discussion of both the visual object store and visual feature store theories has exclusively been about the manner in which information is encoded and retained over the short term. One notable trend is witnessed in the several attempts to try to adjudicate between the theories on the basis of whether attention is necessary for maintaining feature bindings in VSTM. For example, Gajewski and Brockmole (2006) used a task in which a memory display containing six colored items was briefly presented, followed by a spatial cue at one of the item locations. Participants were instructed to make reports about the item, indicated by a response cue that followed the spatial cue. In different conditions, participants reported the shape, the color, or both the color and the shape of the item, as indicated by the response cue. In cases where the spatial cue misdirected attention from the position of the response cue, the profile of reports was well described by a model based on the visual object store theory. There was a propensity for correct reports of both features as well as of neither of the two features, and the incidence of singlefeature reports was actually lower than would be predicted by the idea that features are retained independently when attention is withdrawn.

The work of Gajewski and Brockmole (2006) and others (Allen, Baddeley, \& Hitch, 2006; Johnson, Hollingworth, \& Luck, 2008; Yeh, Yang, \& Chiu, 2005) has provided little support for the framework advanced by Wheeler and Treisman (2002; but see Olson \& Jiang, 2002, for some caveats on the visual object store account). Whereas the cited work examined the role of attention in feature binding, it did not concern the presence and form of the dimensional interactions in VSTM. To this end, we address issues concerning retrieval from VSTM: We ask whether the operations concerning retrieval can be used to help adjudicate between the two theories.

According to the visual feature store theory, an object's features are held independently, such that each different kind of feature is stored in a "dimension-specific cache" colors are stored together, as are shapes, and so forth. The representation of an object is only recovered once focused attention links the corresponding features across the different caches. It is also claimed that, within the separate caches, there is competition for resources, but that there is no such competition between the different caches. Thus, visual feature store theory predicts featural independence during retrieval from VSTM when features on different dimensions are involved.

In contrast, according to visual object store theory, features that are encoded together are stored together. Therefore, feature interactions during encoding provide a mechanism through which partial feature binding can occur without the use of attentional resources. Thus, visual object store theory makes the strong prediction that feature interactions present during encoding should also be evident in retrieval from VSTM. To adjudicate between the two theories, one must assess featural interactions during encoding and then, separately, during retrieval, and compare the two.

In this regard, Cohen (2003) has published a detailed description of the featural interactions between the color and shape of an object during encoding. At the start of a block of trials, Cohen asked observers to judge, on each trial, whether or not each of a designated pair of target features (a color and a shape) was present in a display. Each display contained four colored shapes and was presented for either $33 \mathrm{~ms}$ or $83 \mathrm{~ms}$. Immediately following the display, the observers were asked to make separate confidence ratings for each target feature. There were five conditions of interest - a form target condition, in which only the target shape was present among the three other display items; a hue target condition, in which only the target color was present; a disparate condition, in which both of the target features were present, but in different display items; a coincident target condition, in which both target features were present in the same display item; and finally, a no-target condition, in which neither target feature was displayed.

The results of this detection experiment clearly revealed interactions between the encoding of color and shape. Reports were more accurate when both features were present in a single item (i.e., in the coincident condition) than when they were associated with two items (in the disparate condition). This evidence was consistent with the idea that object-based representations, which code color/shape bindings, are recovered by the perceptual system. 
Further detailed analyses within the context of general recognition theory (GRT; Ashby, 1989, 1992; Ashby \& Townsend, 1986; Kadlec \& Hicks, 1998) have provided additional support for this idea. GRT allows for the precise prediction of participants' sensitivity to the detection of a target, defined by a conjunction of features, from their sensitivity to each individual feature, assuming perceptual and decisional independence. These predictions were not supported. Hence, it was concluded that, during the earliest stages of processing, very close synergies exist in processing color and shape. This is the pattern of results one would predict if the visual system were optimized to process objects (see Duncan, 1984; Vecera \& Farah, 1994; see also Awh, Dhaliwal, Christensen, \& Matsukura, 2001).

The present experiment was undertaken to examine whether or not such dependencies are preserved in VSTM. To this end, a recognition experiment was designed to parallel the earlier detection experiment. The two major changes were that (1)the display duration was increased (to $250 \mathrm{~ms}$ ) and (2)observers did not know which features were to be tested until a probe display was presented. The probe display contained a single, colored shape, and separate yes/no judgments were made for the color and the shape of the probe. The same five conditions employed by Cohen (2003) were used here. If features are stored independently in VSTM, as predicted by the visual feature store theory, the results of the present experiment should show independent retrieval of features (i.e., performance in the coincident condition should equal that in the disparate condition). In contrast, if visual objects are stored in VSTM, as predicted by the visual object store theory, the results of the present experiment should show featural interactions in the retrieval of features.

\section{Method}

Participants

A group of 26 participants were tested from the undergraduate participant panel at the University of York. One participant was removed after a failure to comply appropriately with the experimental instructions. Of the remaining 25 participants, 9 were male, and 23 were right-handed. All reported having normal or corrected-to-normal vision.

\section{Materials and design}

The experiment was controlled by E-Prime software (Schneider, Eschman, \& Zuccolotto, 2002). Each memory display comprised a row of four colored shapes, as shown in Fig. 1. On each trial, four shapes and four colors were sampled at random, without replacement, from the basic eight shapes and eight colors shown in panel a. Each shape was a character from the SPSS Marker Set font (18-point font size).

The probe display contained a single, colored shape positioned centrally. Beneath this shape were four rectangular, labeled click boxes. The boxes were displayed at the four corners of a virtual rectangle. The left boxes registered shape responses, and the right registered color responses. Starting at the upper left box and moving counterclockwise, the boxes were labeled "Shape Yes?", "Shape No?", "Colour No?", and "Colour Yes?". Participants were required to make one "Shape" response and one "Colour" response on every trial by clicking the appropriate boxes.

On target-absent trials, neither the color nor the shape of the probe had been present in the memory display. The four kinds of target-present trials mapped onto those used by Cohen (2003; see above). For ease of exposition, the shapeonly and color-only conditions will be referred to as the single-target conditions, and the coincident and disparate conditions will be referred to as the dual-target conditions.

In the experiment proper, an initial block of 32 practice trials was run. These were 4 target-present trials for each of the four conditions (i.e., shape only, color only, disparate, and coincident), and a corresponding set of 16 target-absent

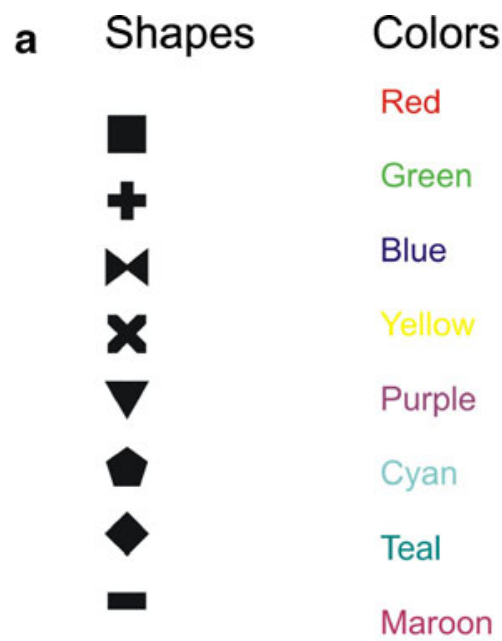

b
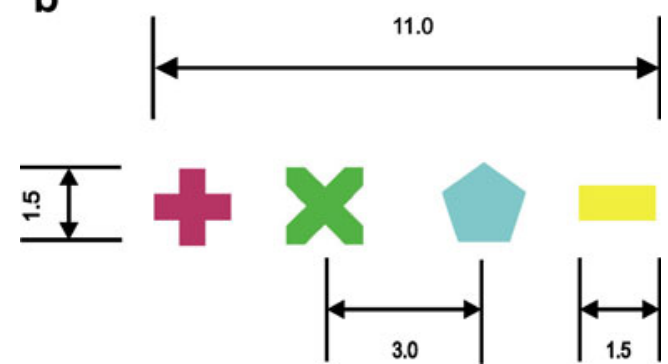

Fig. 1 a The eight shapes and eight colors as used in the experiment. Color names are defined by reference to Windows colors. b An example of a possible sample display with corresponding dimensions, in units of visual angle 
trials. There then followed four blocks of experimental trials. Each block of experimental trials contained 80 targetpresent trials, which were drawn from all four of the conditions, and 80 target-absent trials. Across the four blocks, there were 80 experimental target-present trials for each of the four conditions. The order of trials within each block was randomized on a participant-by-participant basis.

\section{Procedure}

Each participant was tested individually in a quiet testing room. The participant sat at a table facing a 17 -in. computer monitor placed on a plinth, so that the center of the screen was at eye level. A computer mouse was placed in front of the screen, and responses were made via mouse clicks. The viewing distance from the screen was constrained to $57 \mathrm{~cm}$ by a chinrest fixed to the edge of the table.

Participants were instructed as to the nature of the task and that accuracy, not the speed of response, was the primary measure of interest. They were instructed that each trial required a "Shape" and a "Colour" response and that the experiment would move on once allowable responses had been made. The order of report was not constrained. The participants were allowed to complete the block of practice trials and were then asked to sign a consent form. At the start of every trial, a central black dot acted as the fixation point and was presented for $500 \mathrm{~ms}$. This was then

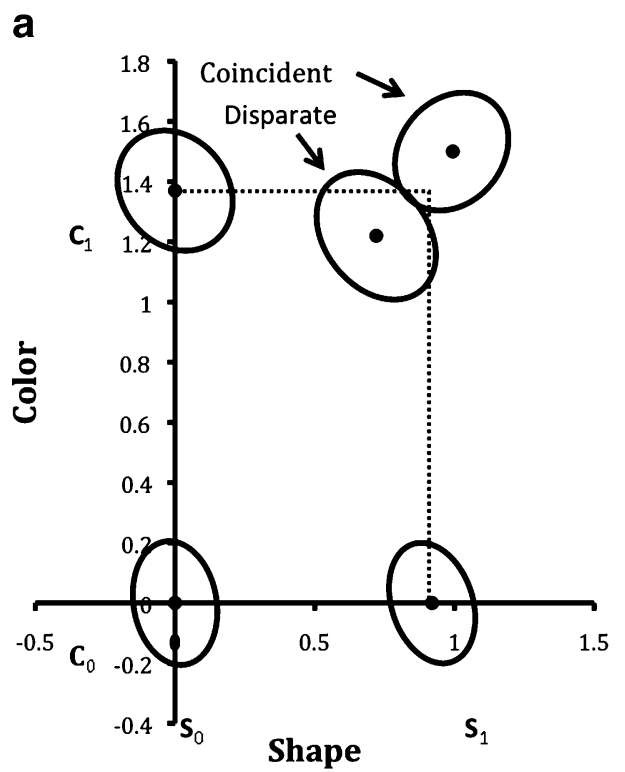

Fig. 2 a Data from the present experiment. bData from Cohen (2003) at the 83-ms display duration. Each panel shows a GRT representation of perceptual effects for the five conditions mapped out in shape $(x) /$ color (y) space. Each distribution is captured by a bivariate mean-a dot - centered within a contour of equal probability. If perceptual separability holds for a dimension, the bivariate mean of a distribution replaced immediately by the sample display, for $250 \mathrm{~ms}$. A blank screen was next presented for $900 \mathrm{~ms}$, and finally the probe display was presented until response. The nature and timing of the displays were based on those used by Allen et al. (2006).

Once two allowable responses had been detected, the fixation dot for the next trial was presented. A rest break was scheduled at the end of each block, and the participant initiated the next block of trials with a press of the mouse.

\section{Results and discussion}

The results of the experiment are shown in Fig. 2 (panel a), together with the results of Cohen (2003) (panel b). The data of interest are the means and distribution of observers' accuracy of report across the five conditions, and these were analyzed according to the various methods set out in GRT. As Fig. 2 shows, performance in the five conditions gives rise to a distribution of points within a 2-D shape/ color space. The points in the space (known as perceptual effects) reflect the evidence in favor of a given state of the world and are partitioned according to the experimental conditions. Each condition distribution is centered on a mean, and the corresponding contour surrounding the mean - a contour of equal likelihood - reflects essentially the dispersion of the corresponding perceptual effects. (The

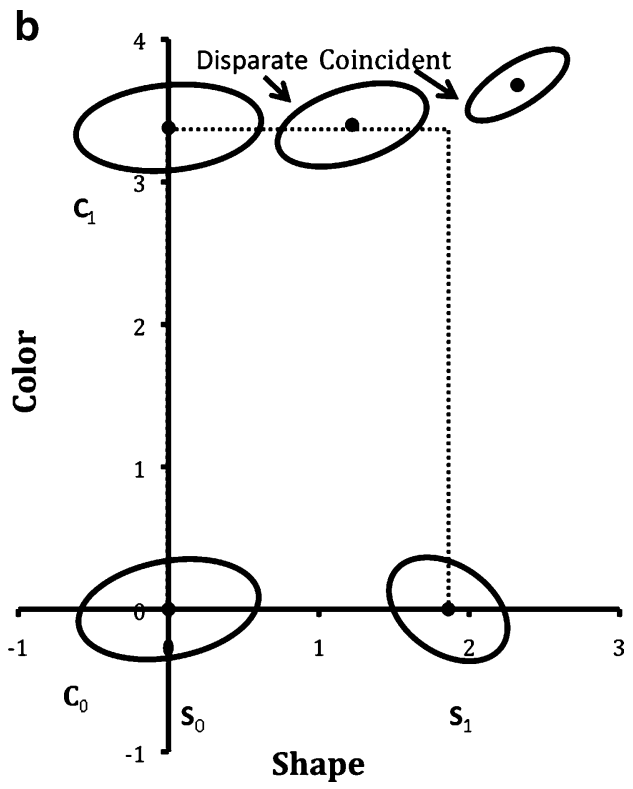

should fall on the dashed line perpendicular to that dimension's axis. With these methods, perceptual separability can only be judged for the disparate and coincident distributions. It fails in both of these cases. This shows that perception of a particular feature value on one dimension depends on the value on the other dimension 
space is viewed "from above," with a section taken through the distributions at an arbitrary height.) The distribution for the no-target condition is fixed at $(0,0)$ in the space, and the other conditions are located relative to this origin. As in standard signal detection theory, the standardized distance between any two means is given by $d^{\prime}$, an index of the sensitivity with which corresponding stimuli can be distinguished. At its most basic level, GRT allows one to identify the presence and form of any interactions between dimensions. In general, GRT makes the same assumptions as signal detection theory (for a review, see Ashby \& Townsend, 1986).

A general impression is that the present tasks are much more difficult than Cohen's (2003). A possible reason for this is that, in Cohen's experiment, each participant searched for the same color and shape probes throughout testing, whereas in the present case, the probe values were taken from a larger sample and changed across the trials. It is also noticeable that reports of color were considerably easier than reports of shape. Without speculating too wildly, this result accords well with color benefits in the data reported by Woodman and Vogel (2008) in similar VSTM tasks.

\section{Marginal d's}

The first test of featural independence assessed what is known as perceptual separability. More particularly, perceptual separability occurs if the perceptual effect of a particular stimulus component is unaffected by the level of the other stimulus component. If color is perceptually separable from shape, then the distribution of percepts (i.e., the impressions of hue) should be the same across the range of shapes tested. Accordingly, impressions of shape should be the same across the range of colors tested. More formally, perceptual separability is violated when the average perceptual effect of one dimension depends on the level of the other dimension. In graphical terms, the critical tests examine the locations of the means of the distributions for the dual-target conditions relative to those in the single-target conditions. According to GRT, perceptual separability holds if the means of the dual-target distributions are fixed at the intersection of the lines perpendicular to each axis extrapolated from the coordinates for the color-only and shape-only means.

In Table 1, the data from the long display duration reported by Cohen (2003; i.e., identification) and the present data (recognition) are presented together. Marginal $d$ 's were calculated on a participant-by-participant basis, and the corresponding means are listed for the single-target and dual-target conditions in the table. Comparisons via paired $t$ tests (two-tailed) were carried out between each single-target condition and each dual-target condition separately. To address problems associated with the family-wise error, a Bonferroni correction was applied, and $\alpha$ was adjusted to .0125 .

The analyses revealed that, relative to the single-target conditions, performance suffered in the disparate condition. Reports of both color and shape were worse when the other probed feature was present in a different display item than when the other probed feature was absent. A contrasting pattern emerged when comparisons were made for the coincident condition. Now color reports were enhanced in the coincident condition relative to the color-only condition. This shows that the presence of the probed shape enhanced reports of the probed color. However, the converse was not found: Shape reports in the coincident condition were no better than reports in the shape-only condition.

\section{Marginal response invariance (MRI)}

A related but different set of analyses confirmed these findings by assessing MRI (see Table 2). MRI holds for one component (say, color) across the different levels of the other component (e.g., shape) if and only if the probability of correctly recognizing the color does not depend on recognizing the shape. From the comparisons between the single- and dual-target conditions, the only case in which MRI is satisfied is where the probabilities of reporting the shape are equivalent across the shape-only and coincident conditions (all other tests, $p<.0125$ ).

Overall, the results of the marginal $d^{\prime}$ and MRI analyses reveal close synergies in the processing of shape and color. When separate features were associated with distinct objects in memory (in the disparate condition), reports of those features suffered relative to when only one target

Table 1 Marginal $d^{\prime}$ analyses

\begin{tabular}{|c|c|c|c|c|c|c|c|c|c|c|}
\hline Task & $\begin{array}{l}\text { Shape } d^{\prime} \\
\text { Shape Rep }\end{array}$ & $\begin{array}{l}\text { Coincident } d^{\prime} \\
\text { rts }\end{array}$ & $p$ & Disparate $d^{\prime}$ & $p$ & $\begin{array}{l}\text { Color } d^{\prime} \\
\text { Color Repc }\end{array}$ & $\begin{array}{l}\text { Coincident } d^{\prime} \\
\text { orts }\end{array}$ & $p$ & Disparate $d^{\prime}$ & $p$ \\
\hline Identification & 1.86 & 2.32 & $<.001$ & 1.22 & $<.001$ & 3.38 & 3.68 & $<.01$ & 3.40 & n.s. \\
\hline Recognition & 0.92 & 0.99 & n.s. & 0.72 & $<.001$ & 1.37 & 1.50 & $<.0125$ & 1.22 & $<.001$ \\
\hline
\end{tabular}

Data for the identification experiment are from Cohen (2003) at the 83-ms display duration. The recognition data are from the present experiment 
Table 2 Marginal response invariance analyses

\begin{tabular}{|c|c|c|c|c|c|c|c|c|c|c|c|c|}
\hline \multirow{3}{*}{ Task } & \multicolumn{6}{|l|}{ Coincident } & \multicolumn{6}{|l|}{ Disparate } \\
\hline & \multicolumn{3}{|c|}{ Shape Reports } & \multicolumn{3}{|c|}{ Color Reports } & \multicolumn{3}{|c|}{ Shape Reports } & \multicolumn{3}{|c|}{ Color Reports } \\
\hline & $p\left(\mathrm{~s}_{1} \mid \mathrm{S}_{1} \mathrm{C}_{0}\right)$ & $p\left(\mathrm{~s}_{1} \mid \mathrm{S}_{1} \mathrm{C}_{1}\right)^{\mathrm{c}}$ & $p$ & $p\left(\mathrm{c}_{1} \mid \mathrm{S}_{0} \mathrm{C}_{1}\right)$ & $p\left(\mathrm{c}_{1} \mid \mathrm{S}_{1} \mathrm{C}_{1}\right)^{\mathrm{c}}$ & $p$ & $p\left(\mathrm{~s}_{1} \mid \mathrm{S}_{1} \mathrm{C}_{0}\right)$ & $p\left(\mathrm{~s}_{1} \mid \mathrm{S}_{1} \mathrm{C}_{1}\right)^{\mathrm{d}}$ & $p$ & $p\left(\mathrm{c}_{1} \mid \mathrm{S}_{0} \mathrm{C}_{1}\right)$ & $p\left(\mathrm{c}_{1} \mid \mathrm{S}_{1} \mathrm{C}_{1}\right)^{\mathrm{d}}$ & $p$ \\
\hline Identification & .88 & .96 & $<.001$ & .94 & .98 & $<.001$ & .88 & .74 & $<.001$ & .94 & .95 & n.s. \\
\hline Recognition & .69 & .71 & n.s. & .60 & .64 & $<.01$ & .69 & .62 & $<.001$ & .60 & .54 & $<.001$ \\
\hline
\end{tabular}

Data for the identification experiment are from Cohen (2003) at the 83-ms display duration. The recognition data are from the present experiment. $p\left(\mathrm{~s}_{1} \mid \mathrm{S}_{1} \mathrm{C}_{0}\right)$ indicates the probability of correctly reporting the shape in the shape condition. $p\left(\mathrm{~s}_{1} \mid \mathrm{S}_{1} \mathrm{C}_{1}\right)^{\mathrm{c}}$ indicates the probability of correctly reporting the shape in the coincident condition. $p\left(\mathrm{c}_{1} \mid \mathrm{S}_{0} \mathrm{C}_{1}\right)$ indicates the probability of correctly reporting the color in the color condition. $p\left(\mathrm{c}_{1} \mid \mathrm{S}_{1} \mathrm{C}_{1}\right)^{\mathrm{c}}$ indicates the probability of correctly reporting the color in the coincident condition. $p\left(\mathrm{~s}_{1} \mid \mathrm{S}_{1} \mathrm{C}_{1}\right)^{\mathrm{d}}$ indicates the probability of correctly reporting the shape in the disparate condition. $p\left(\mathrm{c}_{1} \mid \mathrm{S}_{1} \mathrm{C}_{1}\right)^{\mathrm{d}}$ indicates the probability of correctly reporting the color in the disparate condition

n.s. stands for not statistically significant at the .05 level

feature was presented. In contrast, when both features were associated with the same object (in the coincident condition), reports of either feature were generally better than when only one target feature was presented. These broad patterns of performance are consistent with those found in Cohen (2003), and taken together, they show that the bindings recovered during perceptual encoding are preserved in VSTM.

\section{Sampling independence}

Within GRT, it is also possible to assess whether the error in the detection of one dimension influences the error in the detection of the other: Are errors in the reports of one dimension correlated with errors in the reports of another? To examine this, further analyses examined sampling independence, which holds for a particular color/shape pairing (e.g., a red triangle) if and only if the probability that both features are reported is equal to the multiple of the probabilities that only one feature is reported-for example, that "red" is reported, regardless of what shape report is made, and that "triangle" is reported, regardless of what color is reported. Can the conjoint reporting of color and shape be accounted for by independent reports of color and of shape?

Sampling independence was assessed via an examination of tetrachoric correlations (Ashby, 1988; see Table 3) and was violated in all cases (all $p \mathrm{~s}<.01$, Bonferroni adjusted). These effects are illustrated graphically in Fig. 2a, where the equal-likelihood contours indicate elliptical distributions in every condition that are oriented away from the $x$ and $y$-axes. The orientation of the major axis reflects the sign of the correlation, and the only positive correlation is in the data for the coincident condition.

These correlative data contrast with those found in the identification experiment reported by Cohen (2003). In the identification experiment (Fig. 2b), reports of color and shape were generally positively correlated (i.e., they reflect the facilitative, cooperative nature of encoding colors and shapes). In contrast, the present results show a competitive relationship between the retrieval of color and of shape. Joint reports of both features were only mutually beneficial when both features were stored in memory in a single, object-based representation. In all other cases, perceived variation in one feature impacted negatively on reports of the other feature. As before, these data are evidence against featural independence in VSTM.

\section{Conclusions}

Collectively, the data from the two studies refute the visual feature store theory. Cohen (2003) demonstrated that features interact during encoding, and the present data reveal that the corresponding bindings of encoded features also manifest in VSTM. Moreover, the present data show that object-based effects are present at retrieval. ${ }^{1}$ It seems, therefore, that the principles of perceptual organization that govern stimulus encoding have important consequences for the storage and eventual recovery of visual information over the short term.

The data suggest mechanisms of retrieval that operate on object-based representations. As such, the retrieval of one of the object's features facilitates the retrieval of the object's other features. That is, the act of retrieving one

\footnotetext{
${ }^{1}$ The synergistic effect at encoding would not have any noticeable influence on the interactive effects we identified in the retrieval process. Once the synergistic effect of encoding occurs, it becomes part of the baseline detectability in the task. Because our single-target conditions would include the benefit from that synergistic encoding process, our predictions of independence would include that benefit as well. Furthermore, because we averaged over objects to assess detectability, any differential accuracy in encoding particular objects by the observers would have no overall influence on the interactive effects identified. Thus, the dependencies identified cannot be explained by a single object being randomly chosen and bound by attention prior to the recall task.
} 
Table 3 Results for analyses concerning sampling independence via tetrachoric correlations

\begin{tabular}{|c|c|c|c|c|c|c|}
\hline \multirow[t]{2}{*}{ Condition } & \multicolumn{3}{|l|}{ Identification } & \multicolumn{3}{|l|}{ Recognition } \\
\hline & $p\left(s_{l} c_{1} \mid S_{i} C_{j}\right)$ & $S E$ & $p$ & $p\left(s_{l} c_{1} \mid S_{i} C_{j}\right)$ & $S E$ & $p$ \\
\hline No target $\left(\mathrm{S}_{0} \mathrm{C}_{0}\right)$ & .24 & .08 & $<.001$ & -.14 & .12 & $<.001$ \\
\hline Shape $\left(\mathrm{S}_{1} \mathrm{C}_{0}\right)$ & -.36 & .19 & $<.001$ & -.25 & .21 & $<.001$ \\
\hline Color $\left(\mathrm{S}_{0} \mathrm{C}_{1}\right)$ & .16 & .18 & n.s. & -.19 & .18 & $<.001$ \\
\hline Disparate $\left(\mathrm{S}_{1} \mathrm{C}_{1}\right)$ & .42 & .25 & $<.001$ & -.30 & .16 & $<.001$ \\
\hline Coincident $\left(\mathrm{S}_{1} \mathrm{C}_{1}\right)$ & .72 & .37 & $<.001$ & .21 & .47 & $<.001$ \\
\hline
\end{tabular}

Data for the identification experiment are from Cohen (2003) at the 83 -ms display duration. The recognition data are from the present experiment. $p\left(s_{l} c_{l} \mid S_{i} C_{j}\right)$ indicates the probability of reporting a color and shape target in the condition specified in the row

n.s. stands for not statistically significant at the .05 level

feature from one dimension facilitated the retrieval of a feature on a different dimension when each feature belonged to the same object. This facilitation is evidenced in the synergistic effect identified in the coincident condition. In contrast, when each of the two features belonged to a different object, featural retrieval was suppressed. This suppression is evidenced in the inhibitory effect identified in the disparate condition. A number of different accounts of retrieval can accommodate our inhibitory evidence from the disparate condition, but at present it is impossible to adjudicate between them. One likely account is that the inhibitory effects arise because of featural mismatches during retrieval. When a single probe produces featural mismatches in two different object-based representations, this inhibition may occur both within and between objects. ${ }^{2}$ Thus, competition at retrieval between different featural dimensions exists, but only when the features belong to different objects.

Our results apparently contrast with those reported by Bundesen, Kyllingsbæk, and Larsen (2003) and Kyllingsbæk and Bundesen (2007). Using a slightly different procedure, they found evidence for independent processing of color and form when participants viewed briefly presented and masked displays containing colored shapes (i.e., letters, in the most-relevant cases). Participants had to verbally report any features that they could from the displays. In cases in which the displays contained two colored letters, reports of color and shape were independent from one another. Similar evidence was found when the display set size was increased to three colored lettershowever, some evidence for object-based effects also emerged (see Kyllingsbæk \& Bundesen, 2007, Exp.3). What this seems to suggest is that object-based effects are

\footnotetext{
${ }^{2}$ An implication is that within-object featural inhibition occurs in the single-target conditions, but since these provide the baseline performance, we are unable to judge whether this is the case on the basis of the data.
}

least likely to emerge when the amount of information to be reported falls well short of the capacity limitations of VSTM. A thorough examination of this possibility should form the basis of future research.

In closing, we conclude that information in VSTM is retained in a way that reflects the perceptual operations that were engaged when the stimuli were encoded. According to Cohen and Kubovy (1999), visual feature binding is fundamentally a preattentive process. VSTM retains the encoded feature bindings that reflect the objects that were actually presented for retention. During encoding, feature dimensions interact in very particular ways, and these interactions go on to determine the manner in which information is retrieved from VSTM. The findings reveal that both storage in and retrieval from VSTM operate on the basis of object-based representations. In sum, our data support the visual object store theory.

Author Note We are grateful for discussions about the general issues with Alan Baddeley and Graham Hitch, and are extremely grateful to $\mathrm{Han} \mathrm{Li}$, who ran the participants.

\section{References}

Allen, R. J., Baddeley, A. D., \& Hitch, G. J. (2006). Is the binding of visual features in working memory resource-demanding? Journal of Experimental Psychology: General, 135, 298-313. doi:10.1037/0096-3445.135.2.298

Ashby, F. G. (1988). Estimating the parameters of multidimensional signal detection theory from simultaneous ratings on separate stimulus components. Perception \& Psychophysics, 44, 195-204.

Ashby, F. G. (1989). Stochastic recognition theory. In D. Vickers \& P. L. Smith (Eds.), Human information processing: Measures, mechanisms, and models (pp. 435-457). Amsterdam: Elsevier Science.

Ashby, F. G. (Ed.). (1992). Multidimensional models of perception and cognition. Hillsdale: Erlbaum.

Ashby, F. G., \& Townsend, J. T. (1986). Varieties of perceptual independence. Psychological Review, 93, 154-179.

Awh, E., Dhaliwal, H., Christensen, S., \& Matsukura, M. (2001). Evidence for two components of object-based selection. Psychological Science, 12, 329-334. doi:10.1111/1467-9280.00360

Bundesen, C., Kyllingsbæk, S., \& Larsen, A. (2003). Independent encoding of colors and shapes from two stimuli. Psychonomic Bulletin \& Review, 10, 474-479.

Cohen, D. J. (2003). Direct estimation of multidimensional perceptual distributions: Assessing hue and form. Perception \& Psychophysics, 65, 1145-1160.

Cohen, D., \& Kubovy, M. (1999). Even feature integration is cognitively impenetrable. The Behavioral and Brain Sciences, $22,371-372$.

Duncan, J. (1984). Selective attention and the organization of visual information. Journal of Experimental Psychology: General, 113, 501-517.

Gajewski, D. A., \& Brockmole, J. R. (2006). Feature bindings endure without attention: Evidence from an explicit recall task. Psychonomic Bulletin \& Review, 13, 581-587.

Johnson, J. S., Hollingworth, A., \& Luck, S. J. (2008). The role of attention in the maintenance of feature bindings in visual short- 
term memory. Journal of Experimental Psychology: Human Perception and Performance, 34, 41-55. doi:10.1037/00961523.34.1.41

Kadlec, H., \& Hicks, C. L. (1998). Invariance of perceptual spaces and perceptual separability of stimulus dimensions. Journal of Experimental Psychology: Human Perception and Performance, $24,80-104$.

Kyllingsbæk, S., \& Bundesen, C. (2007). Parallel processing in a multifeature whole-report paradigm. Journal of Experimental Psychology: Human Perception and Performance, 33, 64-82. doi:10.1037/0096-1523.33.1.64

Luck, S. J., \& Vogel, E. K. (1997). The capacity of visual working memory for features and conjunctions. Nature, 390, 279-281. doi: $10.1038 / 36846$

Olson, I. R., \& Jiang, Y. (2002). Is visual short-term memory object based? Rejection of the "strong-object" hypothesis. Perception \& Psychophysics, 64, 1055-1067.

Schneider, W., Eschman, A., \& Zuccolotto, A. (2002). E-Prime user's guide. Pittsburgh: Psychology Software Tools Inc.
Vecera, S. P., \& Farah, M. J. (1994). Does visual attention select objects or locations? Journal of Experimental Psychology: General, 123, 146-160.

Vogel, E. K., Woodman, G. F., \& Luck, S. J. (2001). Storage of features conjunctions and objects in working memory. Journal of Experimental Psychology: Human Perception and Performance, 27, 92-114.

Wheeler, M. E., \& Treisman, A. M. (2002). Binding in short-term memory. Journal of Experimental Psychology: General, 131, 48-64.

Woodman, G. F., \& Vogel, E. K. (2008). Selective storage and maintenance of an object's features in visual working memory. Psychonomic Bulletin \& Review, 15, 223-225. doi:10.3758/ PBR.15.1.223

Yeh, Y.-Y., Yang, C.-T., \& Chiu, Y.-C. (2005). Binding or prioritization: The role of selective attention in visual shortterm memory. Visual Cognition, 12, 759-799. doi:10.1080/ 13506280444000490

Zhang, W., \& Luck, S. J. (2008). Discrete fixed-resolution representations in visual working memory. Nature, 453, 233-236. doi:10.1038/nature06860 